\title{
Marine accident learning with fuzzy cognitive maps (MALFCMs) and Bayesian networks
}

\author{
Beatriz Navas de Maya ${ }^{1}$ (D) Ahmed O. Babaleye ${ }^{1} \cdot$ Rafet E. Kurt $^{1}$
}

Received: 17 June 2019 / Revised: 14 August 2019 / Accepted: 15 August 2019 / Published online: 25 October 2019

(C) The Author(s) 2019

\begin{abstract}
Addressing safety is considered a priority starting from the design stage of any vessel until end-of-life. However, despite all safety measures developed, accidents are still occurring. This is a consequence of the complex nature of shipping accidents where too many factors are involved including human factors. Therefore, there is a need for a practical method, which can identify the importance weightings for each contributing factor involved in accidents. As a result, by identifying the importance weightings for each factor, risk assessments can be informed, and risk control options can be developed and implemented more effectively. To this end, Marine Accident Learning with Fuzzy Cognitive Maps (MALFCM) approach incorporated with Bayesian networks (BNs) is suggested and applied in this study. The MALFCM approach is based on the concept and principles of fuzzy cognitive maps (FCMs) to represent the interrelations amongst accident contributor factors. Thus, MALFCM allows identifying the importance weightings for each factor involved in an accident, which can serve as prior failure probabilities within BNs. Hence, in this study, a specific accident will be investigated with the proposed MALFCM approach.
\end{abstract}

Keywords Maritime accidents $\cdot$ Maritime safety $\cdot$ Maritime accident learning with fuzzy cognitive maps (MALFCMs) $\cdot$ Human factors $\cdot$ Bayesian networks (BNs)

\section{Introduction}

The analysis of historical accident data has revealed that maritime accidents have been traditionally a concern for the shipping industry, as they incur into significant economic consequences, social, and environmental impact (Eliopoulou et al. 2016). Therefore, aiming to reduce the accident rate, maritime organizations are directing efforts into the continuous development and implementation of safety measures, which overall aim to improve maritime safety significantly. Nevertheless, despite all the efforts, maritime accidents are still happening and they remain a major concern when around $90 \%$ of world trading is still carried out by shipping companies (Chauvin et al. 2013).

Moreover, due to inconsistent methods followed during accident investigations, and the additional complexity of identifying all the variables involved into an accident

Beatriz Navas de Maya

beatriz.navas-de-maya@strath.ac.uk

1 University of Strathclyde, 100 Montrose St, Glasgow, G4 0LZ, UK scenario, it is extremely challenging to integrate lessons learnt from past accidents into safety assessments. According to Kristiansen (2013) there is no clear answer to why accidents happen., as they are complex processes, in which there is no a single factor solely responsible for the accident outcome. However, if it is possible to identify and cleverly measure accident-contributing factors, efforts can be focused on addressing these factors to reduce the accidents rate and therefore improve safety.

When analyzing the literature, regardless of the industry in scope, it becomes evident that humans have a significant role into past accidents. For instance, human errors are responsible for at least $66 \%$ of the accidents in strategic sectors such as nuclear and aerospace. In addition, they account for more than $80 \%$ of accidents within the maritime industry (Graziano et al. 2016; Islam et al. 2018; Kurt et al. 2016; Turan et al. 2016).

Hence, this paper aims to identify and weight the importance of each human factor that contributes to the development of past maritime accidents. Thus, this paper applies a new Fuzzy Cognitive Map (FCM)-based technique, Marine Accident Learning with Fuzzy Cognitive Maps (MALFCMs), incorporates BNs to reassess the accident model, and demonstrates it through a case study. By applying MALFCM 
method, it will be possible to weight the individual importance of each human factor from the system under study. Furthermore, this paper proposes to use the information provided by MALFCM as an input to create a Bayesian Network (BN) model. By creating above-mentioned model, it would be possible to study how the accident probabilities might change by addressing some specific human factors (i.e. it would allow to study the system from a "what-if" perspective).

By following the proposed approach, it will be possible to understand the importance of each human factor in maritime accidents, which would allow researchers to incorporate these factors into risk assessments in a more effective way. Thus, by studying the model from a what-if perspective, it would be possible to inform risk assessments and predict the effectiveness of risk control options. The MALFCM results can then be feed into a Bayesian network (BN), which can update and adapt the failure model response as the knowledge of the entire human factor risks become apparent and available. The $\mathrm{BN}$ has a robust computation engine that can provide real-time information about the MALFCM results.

This paper is structured as follows: First, a literature review is provided regarding the FCM method, comprising mainly its mathematical representation, main areas of application and its practical limitation, which is overcome by applying MALFCM method. Thus, this section also provides a comprehensive review of the BN method. In addition, in "Fuzzy cognitive maps (FCMs) theory" a detailed description of the methodology is provided. Moreover, in "Bayesian network $(\mathrm{BN}) "$ the results and discussion are shared. Finally, the conclusions, limitations and recommendations for further work are included in "Methodology".

\section{Fuzzy cognitive maps (FCMs) theory}

When analyzing a complex accident scenario, one of the main challenges lays in the process of classifying the factors involved in it (Wolpert 1992). From the extensive list of available classification methods, FCMs present a set of advantages. First, FCMs allow to model causal relationships between accident variables (Kardaras and Karakostas 1999; M. Khan et al. 2001). Second, an FCM is a suitable technique to represent hazy degrees of causality relations between components (S. Lee and Han 2000). Third, they are considered traditionally as a powerful tool for modelling systems that cannot be explained entirely mathematically (Stylios and Groumpos 1999). In addition, vector-matrix operations allow an FCM model to become a dynamic system (M. Khan et al. 2001; B Kosko 1994) by allowing the system to evolve with time.

By definition FCMs are extension of cognitive maps, in which the main difference with traditional cognitive maps is that the concepts represented in an FCM are weighted (Bart Kosko 1986). Thus, FCMs aim to model complex chains of casual relationships and they have become a potential tool for modelling and analyzing dynamic interactions between concepts or systems in the past years (K. Lee et al. 1996).

For the construction of an FCM model, experts of a specific area of knowledge develop a model based on their experience in a process composed by three stages. First, key concepts are identified within a determined area. Second, interrelationships are proposed between these concepts, identifying if these relations are positive or negative, while in the last step experts estimate the causal relationship strength (Papageorgiou 2010; Zare Ravasan and Mansouri 2016). In terms of decision support, there are two methods to analyze an FCM model. Firstly, a static analysis can be carried out in order to establish the relative importance of concepts and the causal effects between nodes (Axelrod 1976; M. S. Khan and Quaddus 2004). Nevertheless, only a dynamic analysis allow to study and explore the impact in the decision process with time (M. S. Khan and Quaddus 2004).

An FCM is mainly characterized by three elements: the characteristics of the system and signed and weighted arcs representing the interrelations within the different elements. The main target in an FCM is to define the relationships between the different concepts represented in the map, understanding the global structure and the dynamics of the system (Azadeh et al. 2014). Figure 1 shows the structure of a traditional FCM.

Moreover, within an FCM, each of the concepts is represented by a number, $A_{i}$, that represents its value within the interval [0,1] (León et al. 2010). It is possible to identify three types of connections between the concepts described in the FCM that represents the nature of their respective influence (Azadeh et al. 2014; León et al. 2010):

(i) The weights between the concepts $C_{i}$ and $C_{j}$ is positive $\left(\mathrm{W}_{\mathrm{ij}}>0\right)$, which means that an increase in the first concept will lead to an increase in the second concept and vice versa.

(ii) The weights between the concepts $C_{i}$ and $C_{j}$ is negative $\left(\mathrm{W}_{\mathrm{ij}}<0\right)$, which means that an increase in the first

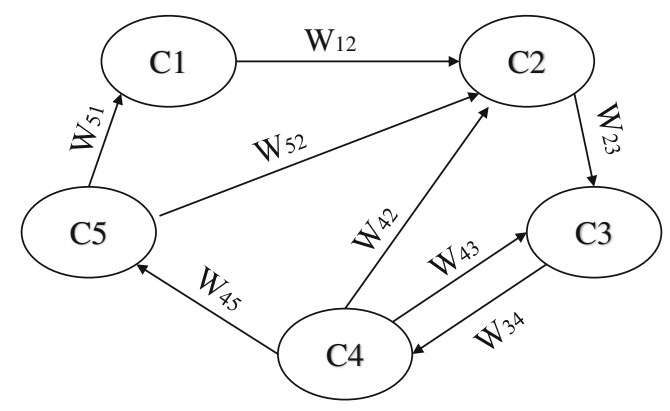

Fig. 1 A simple representation of an FCM, where C represents a concept and $\mathrm{W}$ denotes the interrelation between each pair of concepts (Navas de Maya et al. 2018) 
concept will lead to a decrease in the second concept and vice versa.

(iii) There is no relation between the concepts $C_{i}$ and $C_{j}$ $\left(\mathrm{W}_{\mathrm{ij}}=0\right)$.

According to (Bart Kosko 1986), a traditional formula to calculate the values of concepts in an FCM is represented in Eq. 1, in which $A_{i}$ represents the value of $C_{i}$; $f$ is the threshold function; $\mathrm{W}_{\mathrm{ji}}$ represents the weight between concepts $\mathrm{C}_{\mathrm{i}}$ and $C_{j}$; and $A_{j}$ is the value of the concept $C_{j}$.

$\mathrm{A}_{\mathrm{i}}^{(\mathrm{t}+1)}=\mathrm{f}\left(\mathrm{A}_{\mathrm{i}}^{(\mathrm{t})}+\sum_{\mathrm{j}=1, \quad \mathrm{j} \neq \mathrm{i}}^{\mathrm{n}} \mathrm{W}_{\mathrm{ji}} \mathrm{A}_{\mathrm{j}}^{(\mathrm{t})}\right)$

In order to create successfully an FCM, it is necessary to define three main components. First, an interaction matrix with dimension $\mathrm{n} \times \mathrm{n}$ where $\mathrm{n}$ indicates the number of concepts modelled within the FCM. Thus, a value of zero in the matrix indicates that a relation does not exist between those two particular elements, while non-zero elements show not only that there is a relation between two elements but also the strength or weight of that relation. Second, an initial state vector, which shows the initial value of the concepts in the scenario being modelled at any point in time (t). Finally, a threshold function which aims to reduce unbounded inputs to a strict range, to maintain the stability of the qualitative model (Mohr 1997). Although there are plenty threshold functions available, the Sigmoid function gives any possible value within the interval [0,1] (Azadeh et al. 2014; Xiao et al. 2012) and it has been proved that using this function provides greater benefits (Bueno and Salmeron 2009).

Regarding the dynamic process of an FCM model, once the process starts, the values of the concepts at each time step (i.e. step 1, step 2 etc.) will be obtained by following Eq. 2, until the process stops. The process might stops at any of the following scenarios (M. Khan et al. 2001; B Kosko 1994; Xiao et al. 2012):

(i) The FCM reaches equilibrium, which occurs when after two consecutive steps repeating the process; both state vectors obtained are identical. In this situation, the simulation stops and the FCM is considered steady.

(ii) The FCM does not produce a stable state vector. as it keeps cycling between a certain number of values, (e.g. $0,0.3,0.5,0,0.3,0.5, \ldots, 0,0.3,0.5)$. This situation is known as the "limit cycle", and it results from a certain combination of weight values when applying an FCM, which drive the map away from reaching equilibrium (Wierzchon 1995). Nevertheless, an alternative solution to avoid aforementioned limit cycle is to apply an hybrid system comprising both, FCMs and genetic algorithms (Mateou and Andreou 2006).

(iii) The FCM does not reach identical values, producing different state vectors for each step. This possibility is known as "chaos" and it can appear in complex scenarios, in which a re-definition of the model would be required to overcome a chaos situation.

Despite of the fact that FCM is not as well-known as other methods (Papakostas et al. 2008; Papakostas et al. 2012), it has been proved to be very promising and worth of further investigation and development (Vergini and Groumpos 2016). Several studies have addressed the application of FCMs as a classification tool in different fields for the past years, proving that FCM is not only a well-validated classification tool but also its effectiveness. Thus, FCMs have been widely used in terms of planning and decision making (Dodurka et al. 2017). Moreover, the interest from both researcher and industry is increasing, and FCM has been successfully apply to the areas of medicine (Papageorgiou and Froelich 2012), control (Papageorgiou et al. 2006), business (Glykas 2013), robotics (Motlagh et al. 2012), environmental science (Kok 2009), education (Yesil et al. 2013), energy efficiency (Mpelogianni et al. 2015) and information technology (Büyüközkan and Vardaloğlu 2012).

Although it has been proved in these studies that an FCM is an alternative and powerful method to model and analyse dynamic interactions between concepts or systems, it has an important limitation. As FCMs are designed to transcribe experts' opinion, its weaknesses lay on the uncertainty related with each expert's response. As a result, an FCM can equally encode the experts' lack of knowledge. Therefore, the reliability of a traditional FCM is linked to the experts' knowledge, background and familiarity with the topic that is being addressed. In order to overcome this limitation, a method for Marine Accident Learning with Fuzzy Cognitive Maps (MALFCMs), which differs from the traditional FCM approach, is proposed and applied in this paper, with the aim to establish weights for human factors involved in maritime accidents successfully. Within this new method, each FCM is developed through establishing relationships between factors from past accident experiences. Therefore, the results from the technique followed in this paper might be considered more objective, as this new approach overcomes the main disadvantage of fuzzy cognitive maps (i.e. the subjective results and knowledge deficiencies between experts). Thus, MALFCM method will be fully explained in the next section of this paper.

\section{Bayesian network (BN)}

Bayesian networks (BNs) are directed acyclic graphs that rely on the strength of Bayes' theorem for assessing accident causations where the causations and their dependencies are all variables of uncertainty. The BNs consist of random variables represented by nodes with causations dependencies depicted by directed arcs linking the nodes. Typically, the BN tend to satisfy the Markov condition where each node is conditionally independent of the collection of all its non-descendant nodes (Du et al. 2015; Neapolitan 2004). The transformation of any 
accident model into BN has been covered in many literature (Abimbola and Khan 2019; Babaleye and Kurt 2019; Babaleye et al. 2019; Islam et al. 2018).

\section{Methodology}

\section{Data selection}

In this study, grounding and stranding accidents involving general cargo vessels between 2011 and 2016 are examined. General cargo vessels, which are usually defined as merchant ships carrying goods and materials from one port to another, were selected for this study due to the higher number of data entries when compared with other vessel categories. The distribution of accident data points regarding the vessel type is outlined in Table 1, in which cargo ships are the vessels with a higher number of accidents registered. Thus, Table 2 provides a further insight into general cargo accidents' outcome, where grounding and stranding accidents were identified as the most common accident type. Therefore, sixteen accidents were examined and analyzed within this study. Thus, MALFCM method was applied to these maritime accidents.

\section{Maritime accident learning with fuzzy cognitive maps (MALFCMs)}

MALFCMs method is a Fuzzy Cognitive Map-based technique, which has been designed to combine expert knowledge with lesson learnt from past accident experiences, aiming to provide more reliable weightings, as the input for the scenario being modelled are partially obtained from real maritime accidents (i.e. the subjective results and knowledge deficiencies between experts might be overcome within this method). Thus, MALFCM method could be described in four main stages:

(i) Historical data analysis stage

(ii) Expert opinions stage

(iii) FCM stage

(iv) Consolidation of results stage

Table 1 Total number of accidents per vessel type

\begin{tabular}{lc}
\hline Vessel type & Number of accidents \\
\hline Cargo ship & 50 \\
Fishing vessel & 34 \\
Passenger ship & 19 \\
Service ship & 19 \\
Recreational craft & 9 \\
Inland waterway vessel & 3 \\
Navy ship & 1 \\
\hline
\end{tabular}

Table 2 Total number of accidents' outcome within cargo ships

\begin{tabular}{ll}
\hline Cargo ship & Number of accidents \\
\hline Grounding/stranding & 16 \\
NA & 12 \\
Collision & 10 \\
Contact & 4 \\
Capsizing/Listing & 2 \\
Flooding/Foundering & 2 \\
Damage to ship or equipment & 1 \\
Fire/Explosion & 1 \\
Hull failure & 1 \\
Loss of control & 1 \\
\hline
\end{tabular}

In the historical data analysis stage, historical data is obtained for the scenario being modelled (e.g. a specific vessel category or an accident outcome), in order to identify which human factors leaded into those accidents. Once all the factors have been identified, each pair of factors is statistically compared to create the interaction matrix described in the previous section. Furthermore, analysis is also performed to establish the initial state vector. To create the interaction matrix for the historical data analysis stage, a comparison between each pair of factors is performed by following two main steps as follows:

(i) First, to determinate the relation between Factor ' $a$ ' and Factor ' $b$ ', the historical accident data is filtered to identify those accidents caused by each of the previous factors. Thus, a second filter is applied to identify those accidents that share both factors as a common accident cause.

(ii) Second, the weight of Factor ' $a$ ' over Factor ' $b$ ' is calculated as the relation between the number of accidents with both factors in common, and the number of accidents which have registered Factor ' $a$ ' as an accident contributing factor. Equation 2 provides a better picture of the process being described.

$W_{F a-F b}=\frac{W_{F a \cap F b}}{W_{F a}}$

Above described steps are repeated in order to obtain the relations and weights of each pair of factors. Moreover, the initial stage vector for each human factor is defined as the relation between the number of accidents, which contains that accident-contributing factor, and the total number of accidents considered for the case study.

In the expert opinion stage, experts are requested to provide their knowledge by comparing each pair of factors that were identified within the previous stage. This rating process might 
be accomplished through numeric values. However, as it is extremely challenging for some expert to assign a number value in specific scenarios, an alternative solution is to apply linguistic variables. The seven variables that are used frequently depending on the problem characteristics are: very very low $<$ very low $<$ low $<$ medium $<$ high $<$ very high $<$ very very high (Markinos et al. 2007). The above-mentioned information is used to define the interaction matrix for the expert opinion stage. Moreover, experts are asked to indicate at which level (within the interval $[0,1]$ ) a factor needs to be active in order to have a minimum contribution into an accident. This information allows to define an initial state vector for the expert opinion stage. In addition, as expertise is established with experience, some experts may be more credible. Hence, it is possible to weight each expert's opinion in order to increase or reduce the importance of their feedback (Kandasamy and Smarandache 2003).

In the FCM stage, the selected threshold function is applied to two sets of data, creating two different FCMs. The first FCM is performed by incorporating the results obtained from the historical data stage, while the second FCM integrates the findings from the expert analysis. For both FCMs, the results are analysed, and the obtained weightings are ranked.

Lastly, in the consolidation of result stage, final weightings for each accident-contributing factor are obtained by performing a sensitivity analysis, which combines the results from both FCMs created in the previous stage.

Although MALFCM is conceptually designed to incorporate together the findings from historical data and expert opinion, it can be perfectly applied exclusively to both, historical data and expert opinion. Therefore, due to the additional difficulty in finding reliable experts with a specific background in human contributing factors into maritime accidents, it was decided that for the purpose of this paper, MALFCM would be only applied to historical accident data. Therefore, expert opinion will not be incorporated into the equation, and the findings from this case study will be purely dependent on the historic data analysis stage.

\section{Human-contributing factors into grounding and stranding accident in general cargo vessels}

As it was indicated previously, within this paper sixteen maritime accidents were scrutinized and analysed, aiming to identify those human factors that had a contribution into grounding and stranding accidents in general cargo vessels. Information regarding aforementioned maritime accidents was obtained from the Marine Accident Investigation Branch (MAIB), and identified human factors were grouped into nine categories based on expert judgement, as shown in Table 3.

\section{Application of MALFCM method}

Once all human-contributing factors were identified, an interaction matrix was created. In order to determine the relationship between each pair of factors to fill the interaction matrix, the two steps described in the previous section were followed. For instance, to determinate the relation between HF2 and HF3, the database was first filtered, resulting in five accidents sharing both human factors as a common cause. In addition, eleven accidents recorded HF2, while eight accidents included HF3. Thus, the interrelation between HF2 and HF3 (i.e. $\mathrm{W}_{2,3}$ in the interaction matrix) would be calculated as the relation between the number of accidents that recorded both human factors in the same accident (five accidents), and the number of accidents that included HF2 $\left(\mathrm{W}_{2,3}=0.455\right)$. Similarly, the relation between HF3 and HF2 would be calculated as the relation between the number of accidents in common and the number of accidents that included HF3 $\left(\mathrm{W}_{3,2}=0.625\right)$. Table 4 shows a representation of the interaction matrix for grounding/stranding accidents in general cargo vessels for the period 2011-2016. In addition, for this case study, the state vector was stablished as the statistical occurrence of each factor within the historical accident database. Thus, Table 5 provides the initial state vector (St.0). In addition, Table 5 also provides the dynamic evolution of the FCM until equilibrium is reached, which occurs before step 8 (St.8).

Figure 2 shows the iterative process followed in MALFCM method until equilibrium is reached. In addition, Table 6 shows the final weightings obtained for all humancontributing factors, after the iteration reaches equilibrium and the simulation stops. The weightings provided by MALFCM are constrained to the interval $[0,1]$. Additionally, these results have been normalized, and the final weightings are also displayed on Table 6, to show the contribution of each human factor into accidents in terms of percentage.

Table 3 Human factors involved into grounding/stranding accidents in general cargo vessels

\begin{tabular}{ll}
\hline No & Human factor \\
\hline HF1 & Improper design, installation and working environment \\
HF2 & Inadequate leadership and supervision \\
HF3 & $\begin{array}{c}\text { Inadequate safety management system: Inadequate procedures or } \\
\text { deviation from Standard Operating Procedure (SOP) }\end{array}$ \\
HF4 & Inadequate safety management system: Substandard monitoring \\
HF5 & Lack of communication and coordination \\
HF6 & Lack of safety culture \\
HF7 & Lack of training \\
HF8 & Lack of, improper or late maintenance \\
HF9 & Unprofessional behavior
\end{tabular}


Table 4 Interaction matrix for grounding/stranding accidents in general cargo vessels. Period 2011-2016

\begin{tabular}{llllllllll}
\hline & HF1 & HF2 & HF3 & HF4 & HF5 & HF6 & HF7 & HF8 & HF9 \\
\hline HF1 & - & 0.000 & 0.000 & 0.000 & 0.000 & 0.000 & 0.500 & 0.500 & 0.500 \\
HF2 & 0.000 & - & 0.455 & 0.091 & 0.182 & 0.091 & 0.727 & 0.000 & 0.455 \\
HF3 & 0.000 & 0.625 & - & 0.000 & 0.000 & 0.000 & 0.750 & 0.000 & 0.125 \\
HF4 & 0.000 & 1.000 & 0.000 & - & 0.000 & 0.000 & 0.000 & 0.000 & 1.000 \\
HF5 & 0.000 & 1.000 & 0.000 & 0.000 & - & 0.000 & 1.000 & 0.000 & 0.000 \\
HF6 & 0.000 & 1.000 & 0.000 & 0.000 & 0.000 & - & 1.000 & 0.000 & 1.000 \\
HF7 & 0.091 & 0.727 & 0.545 & 0.000 & 0.182 & 0.091 & - & 0.000 & 0.455 \\
HF8 & 1.000 & 0.000 & 0.000 & 0.000 & 0.000 & 0.000 & 0.000 & - & 0.000 \\
HF9 & 0.125 & 0.625 & 0.125 & 0.250 & 0.000 & 0.125 & 0.625 & 0.000 & -
\end{tabular}

\section{Application of Bayesian networks}

\section{Case study}

The occurrence or non-occurrence of a grounding or stranding accident for a ship is driven by many factors. Such factors rely heavily on human interference to avert or mitigate associated risks. For instance, Improper design, installation and working environment can have significant impact on the steering capability of the ship and on the reliability of on-board equipment. For the on-board equipment to fail, Inadequate safety management system (Substandard monitoring) must have been experienced. The seakeeping (or mooring) ability of the ship can be weakened due to equipment failure and lack of, improper or late maintenance occurring simultaneously. The technical failure due to the loss of major machinery and redundancies can be exacerbated by the mooring failure or loss of navigation. Navigation can be lost if any of SOP (HF3), communication and coordination (HF5), training (HF7), and professional behaviour (HF9) is lacking. The ship is susceptible to grounding or stranding where either of the technical failure, Inadequate leadership and supervision (HF2) and the lack of safety culture (HF6) are a norm rather than exception.

\section{Problem formulation}

Following the estimation of the HF weightings from previous section, first these HF weightings are converted into prior failure probabilities as shown in Table 7. In addition, the prior failure probabilities are fed into the causation nodes of the BN. As can be seen from Fig. 3, the wreck and/or stranding occurrence probability can be estimated. In the presence of new evidence, the interactions of the HFs can be ascertained through experiential learning. To do this, a forward or backward propagation of the accident model is performed, such that, the grounding (GND) node is latched at a pre-defined state to obtain posterior probabilities for the causation nodes, in the backward propagation analysis. In addition, any or all the causation nodes may be latched to a given state, say failed state to obtain new information of the GND occurrence probability.

Through backward propagation analysis, a new evidence, 'failed state' is set for the grounding (GND) occurrence to obtain new information for the causations. These occurrence probabilities are the posterior failure for the causation events (Table 8, column 3). Through the importance measure depicted in Table 8 , the posterior to prior probability ratios increase considerably with HF2 and HF6 being the most probable causes of grounding/stranding by over $500 \%$. To a lesser degree, HF7 and HF1 both contributed to the overall failure by $20 \%$ and 5\%, respectively. However, HF5 and HF8 decrease by $2 \%$ and $3 \%$ indicating that their occurrence probabilities were overestimated during the risk-capturing phase.

\section{Results and discussion}

For the case study presented in this paper, human-contributing factors involved in grounding and stranding accidents in general cargo vessels were identified and weighted, aiming to use them as an input for the BN model. The data that was utilized within the MALFCM method was obtained from an historical
Table 5 State vector and calculation of steady state for grounding/stranding accidents in general cargo vessels. Period 2011-2016

\begin{tabular}{llllllllll}
\hline & HF1 & HF2 & HF3 & HF4 & HF5 & HF6 & HF7 & HF8 & HF9 \\
\hline St.0 & 0.12500 & 0.68750 & 0.50000 & 0.06250 & 0.12500 & 0.06250 & 0.68750 & 0.06250 & 0.50000 \\
St.1 & 0.54674 & 0.79819 & 0.67918 & 0.54674 & 0.56218 & 0.54674 & 0.80807 & 0.51562 & 0.71859 \\
St.2 & 0.66349 & 0.95759 & 0.70959 & 0.56272 & 0.57250 & 0.55869 & 0.94889 & 0.56792 & 0.93869 \\
St.3 & 0.68385 & 0.96814 & 0.74463 & 0.57974 & 0.58580 & 0.57216 & 0.96367 & 0.58218 & 0.95125 \\
St.4 & 0.68755 & 0.97064 & 0.74737 & 0.58074 & 0.58692 & 0.57310 & 0.96633 & 0.58466 & 0.95456 \\
St.5 & 0.68822 & 0.97089 & 0.74793 & 0.58100 & 0.58715 & 0.57332 & 0.96665 & 0.58511 & 0.95488 \\
St.6 & 0.68833 & 0.97094 & 0.74799 & 0.58103 & 0.58717 & 0.57334 & 0.96670 & 0.58519 & 0.95494 \\
St.7 & 0.68835 & 0.97094 & 0.74800 & 0.58103 & 0.58717 & 0.57335 & 0.96671 & 0.58520 & 0.95495 \\
St.8 & 0.68836 & 0.97094 & 0.74801 & 0.58103 & 0.58718 & 0.57335 & 0.96671 & 0.58520 & 0.95495 \\
\hline
\end{tabular}

Once the interaction matrix and the state vector have been defined, the FCM is created by applying Eq. 1, until equilibrium is reached (i.e. before St.8) 


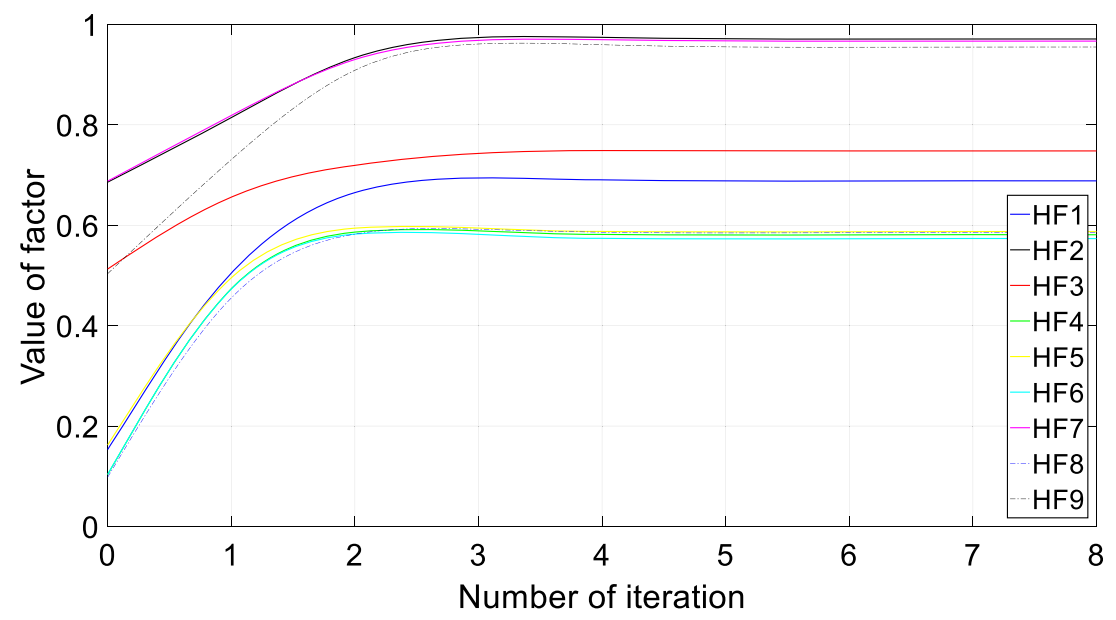

Fig. 2 Values of MALFCM for grounding/stranding accidents in general cargo vessels until equilibrium is reach. Period 2011-2016

accident database for the period 2011-2016. Within this period, sixteen accidents were selected and further analyzed, as they reported human factors that lead into those accidents.

After applying MALFCM method, an "inadequate leadership and supervision" was identified as the most critical factor. An inadequate supervision has been extensively identified in the literature as highly related with maritime accidents. For instance, B. M. Batalden and Sydnes (2017) applied a modified Human Factor Analysis and Classification System (HFACS) framework, identifying that the main causal factors leading to very serious accidents are found in the higher levels of organization, that is organizational influence and unsafe supervision. Thus, Batalden and Sydnes (2014) also performed a study to investigate casualties and incidents, revealing that unsafe supervision emerges as the biggest challenge, and it is a causal factor leading to very serious accidents (34.7\% of analyzed cases), to serious accidents $(23.1 \%)$, and to less serious accidents

Table 6 Final weights of human contributors for grounding/stranding accidents in general cargo vessels. Period 2011-2016

\begin{tabular}{lll}
\hline Human factors & Weight from MALFCM & Weight normalized (\%) \\
\hline HF1 & 0.688 & 10.342 \\
HF2 & 0.971 & 14.588 \\
HF3 & 0.748 & 11.239 \\
HF4 & 0.581 & 8.730 \\
HF5 & 0.587 & 8.822 \\
HF6 & 0.573 & 8.614 \\
HF7 & 0.967 & 14.525 \\
HF8 & 0.585 & 8.793 \\
HF9 & 0.955 & 14.348 \\
\hline
\end{tabular}

(42.1\%). Furthermore, a study conducted by Macrae (2009) on grounding and collision accidents revealed that a lack of supervision and team communication were reported as important contributing factors in the study of grounded vessels.

Moreover, also a "Lack of training" was identified as an important human factor leading into maritime accidents. This observation is in line with previous studies. For example, a study performed by Puisa et al. (2018) revealed that an inadequate training was observable in numerous past accidents, and it was a frequent causal factor across all reports analyzed within aforementioned study. In addition, Graziano et al. (2016) applied the Technique for Retrospective and Predictive Analysis of Cognitive Errors (TRACEr), finding that most of the

Table 7 Process to estimate HF prior failure probabilities from $\mathrm{HF}$ weightings

\begin{tabular}{|c|c|c|c|c|c|}
\hline $\mathrm{HF}$ & $\begin{array}{l}\text { Weights } \\
\text { normalized } \\
(\%)\end{array}$ & $\begin{array}{l}\text { Wrek/ } \\
\text { stranding } \\
\text { frequency } \\
\text { (per ship } \\
\text { year) } \\
\text { (IMO) }\end{array}$ & $\begin{array}{l}\text { Human factor } \\
\text { contribution } \\
\text { into accidents } \\
\text { (estimation } \\
\text { from literature } \\
\text { review) }\end{array}$ & $\begin{array}{l}\text { Wrek/ } \\
\text { stranding } \\
\text { frequency } \\
\text { (per ship } \\
\text { year) due } \\
\text { to HF }\end{array}$ & $\begin{array}{l}\text { Wrek/ } \\
\text { stranding } \\
\text { frequency } \\
\text { of each HF } \\
\text { (per ship } \\
\text { year) }\end{array}$ \\
\hline HF1 & 10.342 & 0.0075 & $80 \%$ & 0.006 & $6.21 \mathrm{E}-04$ \\
\hline HF2 & 14.588 & & & & $8.75 \mathrm{E}-04$ \\
\hline HF3 & 11.239 & & & & $6.74 \mathrm{E}-04$ \\
\hline HF4 & 8.730 & & & & $5.24 \mathrm{E}-04$ \\
\hline HF5 & 8.822 & & & & $5.29 \mathrm{E}-04$ \\
\hline HF6 & 8.614 & & & & $5.17 \mathrm{E}-04$ \\
\hline HF7 & 14.525 & & & & $8.72 \mathrm{E}-04$ \\
\hline HF8 & 8.793 & & & & $5.28 \mathrm{E}-04$ \\
\hline HF9 & 14.348 & & & & $8.61 \mathrm{E}-04$ \\
\hline
\end{tabular}




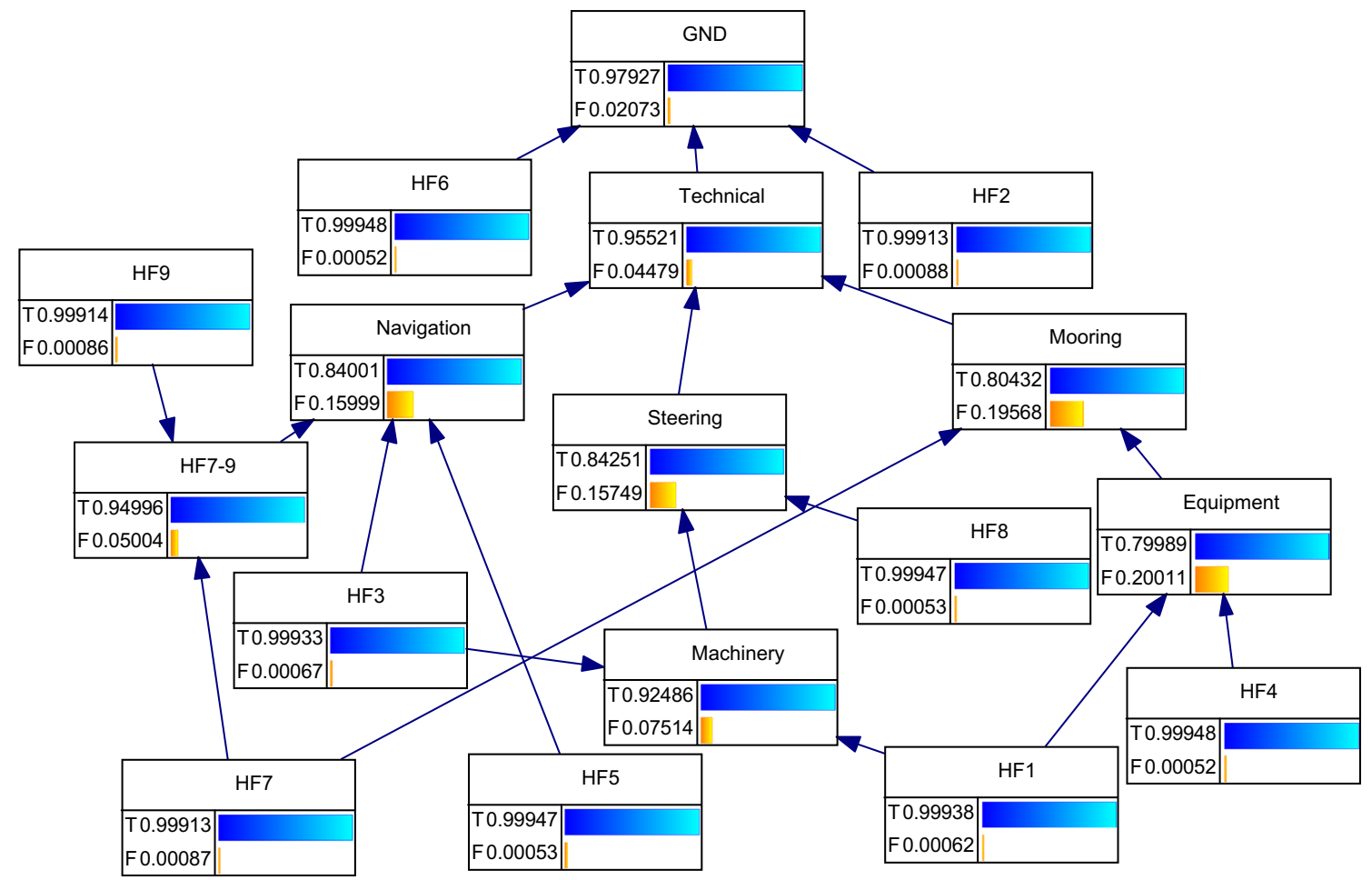

Fig. $3 \mathrm{BN}$ model for the wreckage/stranding accident

failures were associated with factors like fatigue or inadequate training/instruction. Thus, a study performed by Kum and Sahin (2015) on arctic regions revealed that maritime accidents on those regions were mainly associated with inadequate quality and extension of training.

Finally, an "Unprofessional behaviour" was identified as the third main contributing factor within this case study as validated by the results obtained from the $\mathrm{BN}$ and MALFCM. Inadequate behaviours have been previously linked to maritime accident in the literature. For example, a study performed by Antão et al. (2008) highlighted that inadequate behaviors are identified within particular tasks, leading into occupational accidents.

\section{Conclusions}

This study investigates the human factors contribution to global maritime accidents, with emphasis on stranding and/ or grounding of ship structures. A new methodology is introduced for modelling the potential causations of maritime accidents using Marine Accident Learning with Fuzzy Cognitive Maps (MALFCM) incorporated with Bayesian networks. The weightings of the accident contributors are estimated based on the application of MALFCM method. These weightings are then fed into the causal nodes of the Bayesian network to systematically evaluate the stranding/grounding occurrence probability. The Bayesian network model is used as a tool to update the occurrence failure probabilities for each
Table 8 Posterior occurrence probabilities for grounding accidents in general cargo vessels

\begin{tabular}{llll}
\hline Human factors & Prior Probability & Posterior Probability & Importance Measure $\left(\mathrm{P}_{\mathrm{f}} / \mathrm{P}_{\mathrm{i}}\right)$ \\
\hline HF1 & $6.21 \mathrm{E}-04$ & $6.50 \mathrm{E}-04$ & 1.05 \\
HF2 & $8.75 \mathrm{E}-04$ & $4.86 \mathrm{E}-03$ & 5.55 \\
HF3 & $6.74 \mathrm{E}-04$ & $6.80 \mathrm{E}-04$ & 1.01 \\
HF4 & $5.24 \mathrm{E}-04$ & $5.28 \mathrm{E}-04$ & 1.01 \\
HF5 & $5.29 \mathrm{E}-04$ & $5.20 \mathrm{E}-04$ & 0.98 \\
HF6 & $5.17 \mathrm{E}-04$ & $2.81 \mathrm{E}-03$ & 5.44 \\
HF7 & $8.72 \mathrm{E}-04$ & $1.05 \mathrm{E}-03$ & 1.20 \\
HF8 & $5.28 \mathrm{E}-04$ & $5.13 \mathrm{E}-04$ & 0.97 \\
HF9 & $8.61 \mathrm{E}-04$ & $8.60 \mathrm{E}-04$ & 1.00 \\
\hline
\end{tabular}


human factor contribution, given that a new evidence become available about the grounding/stranding accident. The developed framework was applied to a case study on grounding/ stranding accidents in general cargo vessels. In the accident scenario analysis, it was observed that the lack of safety culture (HF6) contributed most to the system failure based on the posterior to prior failures ratio. Based upon the prior failure probabilities obtained from the MALFCM model analysis, it was seen that Inadequate leadership and supervision (HF2), lack of training (HF7) and unprofessional behavior (HF9) are the most probable causes of stranding and/or grounding accident.

Acknowledgements The authors of this paper would like to acknowledge the support of the Marine Accident Investigation Branch (MAIB) who provided the required data for performing this study.

\section{Compliance with ethical standards}

Conflict of interest On behalf of all authors, the corresponding author states that there is no conflict of interest.

Open Access This article is distributed under the terms of the Creative Commons Attribution 4.0 International License (http:// creativecommons.org/licenses/by/4.0/), which permits unrestricted use, distribution, and reproduction in any medium, provided you give appropriate credit to the original author(s) and the source, provide a link to the Creative Commons license, and indicate if changes were made.

\section{References}

Abimbola M, Khan F (2019) Resilience modeling of engineering systems using dynamic object-oriented Bayesian network approach. Comput Ind Eng 130:108-118

Antão P, Almeida T, Jacinto C, Guedes Soares C (2008) Causes of occupational accidents in the fishing sector in Portugal. Saf Sci 46(6): 885-899. https://doi.org/10.1016/j.ssci.2007.11.007

Axelrod, R. M. (1976). Structure of Decision: The Cognitive Maps of Political Elites (R. Axelrod Ed.): Princeton University press

Azadeh A, Salehi V, Arvan M, Dolatkhah M (2014) Assessment of resilience engineering factors in high-risk environments by fuzzy cognitive maps: A petrochemical plant. Saf Sci 68:99-107. https://doi. org/10.1016/j.ssci.2014.03.004

Babaleye AO, Kurt RE (2019) Safety analysis of offshore decommissioning operation through Bayesian network. Ships Offshore Structures:1-11

Babaleye AO, Kurt RE, Khan F (2019) Safety analysis of plugging and abandonment of oil and gas wells in uncertain conditions with limited data. Reliability Engineering System Safety 188:133-141

Batalden B-M, Sydnes AK (2014) Maritime safety and the ISM code: a study of investigated casualties and incidents 13(1):3-25. https:// doi.org/10.1007/s13437-013-0051-8

Batalden, B. M., \& Sydnes, A. K. (2017). What causes 'very serious' maritime accidents? Safety and Reliability - Theory and Applications - epin \& Briš (Eds) (C) 2017 Taylor \& Francis Group, London, ISBN 978-1-138-62937-0

Bueno S, Salmeron JL (2009) Benchmarking main activation functions in fuzzy cognitive maps. Expert Syst Appl 36(3):5221-5229
Büyüközkan G, Vardaloğlu Z (2012) Analyzing of CPFR success factors using fuzzy cognitive maps in retail industry. Expert Syst Appl 39(12):10438-10455

Chauvin C, Lardjane S, Morel G, Clostermann J-P, Langard B (2013) Human and organisational factors in maritime accidents: analysis of collisions at sea using the HFACS. Accid Anal Prev 59:26-37. https://doi.org/10.1016/j.aap.2013.05.006

Dodurka MF, Yesil E, Urbas L (2017) Causal effect analysis for fuzzy cognitive maps designed with non-singleton fuzzy numbers. Neurocomputing 232:122-132. https://doi.org/10.1016/j.neucom. 2016.09.112

Du S, Yao X, Huang D (2015) Engineering model-based Bayesian monitoring of ramp-up phase of multistage manufacturing process. Int J Prod Res 53(15):4594-4613

Eliopoulou E, Papanikolaou A, Voulgarellis M (2016) Statistical analysis of ship accidents and review of safety level. Saf Sci 85:282-292. https://doi.org/10.1016/j.ssci.2016.02.001

Glykas M (2013) Fuzzy cognitive strategic maps in business process performance measurement. Expert Syst Appl 40(1):1-14

Graziano A, Teixeira AP, Guedes Soares C (2016) Classification of human errors in grounding and collision accidents using the TRACEr taxonomy. Saf Sci 86:245-257. https://doi.org/10.1016/j.ssci.2016. 02.026

Islam R, Khan F, Abbassi R, Garaniya V (2018) Human error probability assessment during maintenance activities of marine systems. Saf Health Work 9(1):42-52

Kandasamy, W. V., \& Smarandache, F. (2003). Fuzzy cognitive maps and neutrosophic cognitive maps: infinite study

Kardaras D, Karakostas B (1999) The use of fuzzy cognitive maps to simulate the information systems strategic planning process. Inf Softw Technol 41(4):197-210

Khan MS, Quaddus M (2004) Group decision support using fuzzy cognitive maps for causal reasoning. Group Decis Negot 13(5):463-480

Khan, M., Quaddus, M., \& Intrapairot, A. (2001). Application of a fuzzy cognitive map for Analysing data warehouse diffusion. Paper presented at the Applied informatics-proceedings

Kok K (2009) The potential of fuzzy cognitive maps for semi-quantitative scenario development, with an example from Brazil. Glob Environ Chang 19(1):122-133

Kosko B (1986) Fuzzy cognitive maps. International journal of manmachine studies 24(1):65-75

Kosko, B. (1994). The new science of fuzzy logic: fuzzy thinking. HarperCollins, LondonLane DC, Oliva R (1998) The greater whole: towards a synthesis of the system dynamics and soft system methodology. Eur J Oper Res, 107(1998), 214235Lee

Kristiansen, S. (2013). Maritime Transportation: Safety Management and Risk Analysis: Taylor \& Francis

Kum S, Sahin B (2015) A root cause analysis for Arctic marine accidents from 1993 to 2011. Saf Sci 74:206-220. https://doi.org/10.1016/j. ssci.2014.12.010

Kurt RE, Khalid H, Turan O, Houben M, Bos J, Helvacioglu IH (2016) Towards human-oriented norms: considering the effects of noise exposure on board ships. Ocean Engineering, 120(supplement C), 101-107. https://doi.org/10.1016/j.oceaneng.2016.03.049

Lee S, Han I (2000) Fuzzy cognitive map for the design of EDI controls. Inf Manag 37(1):37-50

Lee K, Kim S, Sakawa M (1996) On-line fault diagnosis by using fuzzy cognitive map. IEICE Trans Fundam Electron Commun Comput Sci 79(6):921-927

León M, Rodriguez C, García MM, Bello R, Vanhoof K (2010) Fuzzy Cognitive Maps for Modeling Complex Systems. In: Fuzzy cognitive maps for modeling complex systems. Paper presented at the Mexican International Conference on Artificial Intelligence

Macrae C (2009) Human factors at sea: common patterns of error in groundings and collisions. Marit Policy Manag 36(1):21-38 
Markinos A, Papageorgiou E, Stylios C, Gemtos T (2007) Introducing fuzzy cognitive maps for decision making in precision agriculture. Precis Agric 7:223

Mateou, N., \& Andreou, A. (2006). An evolutionary methodology to eliminate the limit cycle phenomenon in fcm-based models. Paper presented at the information and communication technologies, 2006. ICTTA'06. 2nd

Mohr, S. (1997). Software design for a fuzzy cognitive map modeling tool. Tensselaer Polytechnic Institute

Motlagh O, Tang SH, Ismail N, Ramli AR (2012) An expert fuzzy cognitive map for reactive navigation of mobile robots. Fuzzy Sets Syst 201:105-121

Mpelogianni V, Marnetta P, Groumpos PP (2015) Fuzzy cognitive maps in the Service of Energy Efficiency. IFAC-PapersOnLine 48(24):16. https://doi.org/10.1016/j.ifacol.2015.12.047

Navas de Maya B, Kurt RE, Turan O (2018) Application of fuzzy cognitive maps to investigate the contributors of maritime collision accidents. Transport Research Arena (TRA) 2018

Neapolitan, R. E. (2004). Learning bayesian networks (Vol. 38): Pearson prentice hall upper Saddle River, NJ

Papageorgiou, E. I. (2010). A novel approach on constructed dynamic fuzzy cognitive maps using fuzzified decision trees and knowledgeextraction techniques. In Fuzzy Cognitive Maps (pp. 43-70): springer

Papageorgiou EI, Froelich W (2012) Multi-step prediction of pulmonary infection with the use of evolutionary fuzzy cognitive maps. Neurocomputing 92:28-35

Papageorgiou EI, Stylios C, Groumpos PP (2006) Unsupervised learning techniques for fine-tuning fuzzy cognitive map causal links. International Journal of Human-Computer Studies 64(8):727-743

Papakostas GA, Boutalis YS, Koulouriotis DE, Mertzios BG (2008) Fuzzy cognitive maps for pattern recognition applications. Int $\mathrm{J}$ Pattern Recognit Artif Intell 22(08):1461-1486
Papakostas GA, Koulouriotis DE, Polydoros AS, Tourassis VD (2012) Towards Hebbian learning of fuzzy cognitive maps in pattern classification problems. Expert Syst Appl 39(12):10620-10629

Puisa R, Lin L, Bolbot V, Vassalos D (2018) Unravelling causal factors of maritime incidents and accidents. Saf Sci 110:124-141

Stylios CD, Groumpos PP (1999) Fuzzy cognitive maps: a model for intelligent supervisory control systems. Comput Ind 39(3):229-238

Turan O, Kurt RE, Arslan V, Silvagni S, Ducci M, Liston P, Schraagen JM, Fang I, Papadakis G (2016) Can we learn from aviation: safety enhancements in transport by achieving human orientated resilient shipping environment. Transportation Research Procedia 14:16691678. https://doi.org/10.1016/j.trpro.2016.05.132

Vergini ES, Groumpos PP (2016) A new conception on the fuzzy cognitive maps method. IFAC-PapersOnLine 49(29):300-304

Wierzchon ST (1995) The fuzzy systems handbook. A practitioner's guide to building, using, and maintaining fuzzy systems: by earl COX; AP professional; Boston, MA, USA; 1994; xxxix+ 624 pp.; \$49-95; ISBN: 0-12-194270-8. Pergamon, In

Wolpert DH (1992) Stacked generalization. Neural Netw 5(2):241-259

Xiao Z, Chen W, Li L (2012) An integrated FCM and fuzzy soft set for supplier selection problem based on risk evaluation. Appl Math Model 36(4):1444-1454

Yesil, E., Ozturk, C., Dodurka, M. F., \& Sahin, A. (2013). Control engineering education critical success factors modeling via Fuzzy Cognitive Maps. Paper presented at the information technology based higher education and training (ITHET), 2013 international conference on

Zare Ravasan A, Mansouri T (2016) A dynamic ERP critical failure factors modelling with FCM throughout project lifecycle phases. Production Planning \& Control 27(2):65-82 